\title{
Professional flossing is effective in reducing interproximal caries risk in children who have low fluoride exposures
}

\author{
Does dental flossing reduce interproximal caries incidence?
}

\section{Hujoel PP, Cunha-Cruz J, Banting DW, Loesche WJ. \\ Dental flossing and interproximal caries: a systematic review. J Dent Res 2006; 85:298-305}

Data sources Searches were made for data using Medline, the Cochrane Central Register of Controlled Trials, Web of Science and the controlled-trials database of clinical trials (www.controlled_trials.com). Reference lists of potentially relevant reports and review articles were also searched. Attempts to obtain missing information and 'grey' literature were made through contact with selected investigators.

Study selection The treatment comparisons of interest included flossing versus no flossing, or a comparison of different frequencies of flossing use. Studies where the effect of flossing could not be separated from the effects of other treatments were excluded. The primary study outcome was a measure of caries incidence. There were no restrictions with respect to the study population. Study designs were limited to controlled clinical trials.

Data extraction and synthesis Quality assessment was performed by evaluating the following items: random allocation, treatment allocation concealment, blinding of outcomes assessors, presentation of point estimates with a measure of variability for the primary outcome measure, 'intention to treat' analysis, report of baseline characteristics by treatment group, eligibility criteria, loss to follow-up, and missing values. For each trial, the number of surfaces at risk and the number of new interproximal caries lesions were derived from or estimated based on published data. Both relative risk (RR) and risk difference and their respective standard deviations were calculated. Heterogeneity was assessed with the $\mathrm{I}^{2}$ statistic (describes the percentage of the variability in effect estimates that is due to heterogeneity rather than sampling error. A value greater than $50 \%$ may be considered substantial heterogeneity). The effect of study characteristics such as fluoride, oral hygiene or caries risk on flossing effectiveness was estimated by meta-regression. Subgroup analyses and sensitivity analyses were also conducted.

Results The search identified 144 articles, of which six met the inclusion criteria. A total of 808 subjects, aged 4 to 13 years, were included in the meta-analysis. There were significant study-to-study differences and a moderate to large potential for bias. Professional flossing performed on school days for 1.7 years on predominantly primary teeth in children was associated with a $40 \%$ caries risk reduction [RR, 0.60; $95 \%$ confidence interval $(\mathrm{Cl}), 0.48-0.76 ; \mathrm{P}<0.001]$. Both 3 -monthly professional flossing for 3 years (RR, $0.93 ; 95 \% \mathrm{Cl}, 0.73-1.19 ; \mathrm{P} 0.32$ ) and selfperformed flossing in young adolescents for 2 years (RR, $1.01 ; 95 \% \mathrm{Cl}$, $0.85-1.20$; P 0.93) did not reduce caries risk. No flossing trials in adults or under unsupervised conditions could be identified.

Conclusions Professional flossing in children with low fluoride exposures is highly effective in reducing interproximal caries risk. These findings should be extrapolated to more typical floss-users with care, since self-flossing has failed to show an effect.

\section{Commentary}

This is an excellent and particularly detailed systematic review of the effect of flossing on interproximal caries. The conclusions are precisely delineated and supported by the evidence. The authors are to be commended on their discussion section, in which they explore the limitations of the results and their implications in terms of research and dental practice. They draw attention to a number of issues, including the lack of anticaries effect seen in self-flossing studies and the potential positive and negative effects on caries of different flossing techniques. They raise the point that the current evidence is unable to answer the question of whether flossing provides a benefit above and beyond brushing with a fluoride toothpaste.

The authors conclude that the current level of evidence is consistent with the hypothesis that regular and meticulous flossing can drastically lower interproximal caries risk in young children who have poor toothbrushing habits and low fluoride exposure.

The evidence-based approach is nested in reductionist science and a corollary to this rigorous paper is: can such methodology cope with a process involving interdependent variables? In this particular case the complexity of the inter-relationship of flossing and fluoride in caries development may be difficult, if not impossible, to delineate. For example, will efficient daily removal of interproximal plaque reduce the anticaries effect of fluoride by attenuating (or even eliminating) the 'fluoride-reservoir' effect of the biofilm? The two effects are inter-related and one cannot vary the one without influencing the effect of the other.

Studies investigating the anticaries effects of combinations of professional cleaning (including flossing) and topical fluorides were quite correctly excluded, considering this systematic review's methodology. In the real world, however, the combination of those two factors is what occurs: everyone who flosses their teeth effectively also uses fluoride toothpaste. Although the authors suggest that studies embedding factorial designs may provide the answers to determining what fraction, if any, of interproximal caries can be prevented by dental floss in a fluoridated world, such studies may require more complex analytical tools than are currently available. It may be impossible to answer that question and, in any case, it may be irrelevant, since, in the real world, flossing is inextricably intertwined with brushing using fluoride toothpaste. Thus, rather than determining the role of flossing per se, it may be more appropriate to try to determine through an RCT if there is any positive or negative impact (on the anti-caries benefit from brushing with a fluoride toothpaste) of a combination of flossing and brushing (with a fluoride toothpaste).

\section{Practice point}

Regular flossing of children's teeth by a trained adult can dramatically reduce interproximal caries in those at high risk of caries.

\section{Chris Longbottom}

Centre for Clinical Innovations, University of Dundee, Dundee, Scotland, UK
Address for correspondence: PP Hujoel, Department of Dental Public Health Sciences, School of Dentistry, Box 357475, University of Washington, Seattle WA 98195, USA. E-mail: hujoel@u.washington.edu. 